\title{
IMPACT OF SELF-SIMILARITY ON WIRELESS DATA NETWORK PERFORMANCE
}

\author{
Michael Jiang, Milan Nikolic, Stephen Hardy, and Ljiljana Trajkovic * \\ School of Engineering Science \\ Simon Fraser University \\ Vancouver, British Columbia, Canada \\ \{zjiang, milan, steve, ljilja\}@cs.sfu.ca
}

\begin{abstract}
In this paper we investigate the impact of traffic patterns on wireless data networks. Modeling and simulation of the Cellular Digital Packet Data (CDPD) network of Telus Mobility (a commercial service provider) were performed using the OPNET tool. We use trace-driven simulations with genuine traffic trace collected from the CDPD network to evaluate the performance of CDPD protocol. This trace tends to exhibit longrange dependent behavior. Our simulation results indicate that genuine traffic traces, compared to traditional traffic models such as the Poisson model, produce longer queues and, thus, require larger buffers in the deployed network's elements.
\end{abstract}

Keywords - wireless networks, mobile networks, CDPD networks, long-range dependence.

\section{INTRODUCTION}

Traffic patterns generated by voice, data, and image services, which are available in current packet switched data networks, differ from patterns observed in circuit switched voice networks. Simulating these services requires traffic models that differ from traditional Poisson models used for voice traffic. Users may experience poor performance due to incorrect traffic assumptions made when provisioning and designing data networks based on traditional traffic models.

Interest in self-similar traffic was first stimulated by the measurements of Ethernet traffic at Bellcore [8]. The analysis of the collected traffic traces led to the discovery that "traffic looks the same on all time scales" $[8,15,16]$ and to the introduction of the term self-similar (or fractal) traffic. Since then, this feature has been discovered in many other traffic traces, such as Transmission Control Protocol (TCP) [10, 11], Motion Pictures Experts Group (MPEG) video [5], World Wide Web [3], and Signaling System 7 [4] traffic. An important characteristic of self-similar traffic is its long-range dependence, i.e., the existence of correlations over a broad range of time scales $[2,12]$.

Most network traffic measurements have been performed on wired networks. The question arises whether the traffic in wireless data networks exhibits self-similar behavior as well. If so, it is important to determine if this traffic characteristic affects the provisioning and design of wireless data networks.
To answer these questions, we analyzed traffic from a Cellular Digital Packet Data (CDPD) [1, 14] network of a mobile data service provider (Telus Mobility). Our initial statistical analysis results indicate that this trace exhibits a certain degree of self-similarity. This long-range dependent behavior is statistically different from the behavior of traffic generated by traditional traffic models. In order to evaluate the performance of CDPD wireless networks, we use the OPNET tool [6] to simulate the Telus Mobility CDPD network. In our simulations, we use genuine traffic trace collected from the Telus Mobility network. Our simulation results indicate that, as a consequence of being long-range dependent, genuine traffic produces longer queues. Hence, it requires larger buffers in the network's elements than traffic generated by traditional models.

The paper is organized as follows. In Section II, we describe wireless traces and provide statistical analysis and the test for self-similarity. In Section III, we describe the CDPD protocol and the OPNET simulation of the CDPD network. Simulation results and implications on traffic modeling are given in Section IV. We conclude with Section V.

\section{TRAFFIC MEASUREMENTS AND ANALYSIS}

\section{A. Self-similar stochastic process and statistical tests}

We present here a brief definition of self-similarity for stochastic processes [2]. Assume $X_{k}(\mathrm{k}=1,2, \ldots)$ to be a wide-sense stationary process with mean $E\left(X_{k}\right)=\bar{X}$ and autocorrelation function $r(i)$. Next, consider the processes $X_{k}^{(m)} \quad(\mathrm{m}=1,2, \ldots)$ that are constructed from $X_{k}$ by averaging over non-overlapping blocks of size $m$ as $X_{k}^{(m)}=\left(\sum_{l=0}^{m-1} X_{k m+l}\right) / m$. The processes $X_{k}^{(m)}$ are also widesense stationary, with mean $\bar{X}$ and autocorrelation function $r^{(m)}(i)$. The process $X_{k}$ is called (exactly) second-order selfsimilar if $r^{(m)}(i)=r(i)$ for $i \geq 0$, and (asymptotically) secondorder self-similar if $r^{(m)}(i)=r(i)$, for $m \rightarrow \infty$.

An important parameter of a self-similar process is the Hurst parameter $H$ that can be estimated from the variance of a

\footnotetext{
* This research was funded in part by Canadian Foundation for Innovation Grant 910-99 and the Grant-in-Aid from Telus Mobility.
} 
statistical process. Self-similarity is implied if $0.5<H<1$. We used two graphical methods to test the self-similarity of the wireless data traffic trace: $R / S$ and variance-time plots [8].

The $R / S$ analysis plot is the rescaled adjusted range plot or the pox diagram of R/S [8]. In Fig. 1, we plot $\log (R(n) / S(n))$ vs. $\log (n)$, where $R(n)$ is the adjusted range statistic of the process and $S(n)$ is sample standard deviation. For a set of observations $X_{k}(\mathrm{k}=1,2, \ldots, \mathrm{n})$, the $\mathrm{R} / \mathrm{S}$ statistic is given by:

$R(n) / S(n)=\left[\max \left(0, W_{1}, W_{2}, \ldots, W_{n}\right)-\min \left(0, W_{1}, W_{2}, \ldots, W_{n}\right)\right] / S(n)$

where $W_{k}=\left(X_{1}+X_{2}+\ldots+X_{k}\right)-k \bar{X}(n)(k \geq 1)$, with sample mean $\bar{X}(n)$. Logarithmically spaced values of $n$ are taken, starting with $n \approx 10$. The slope of the coned zone with plotted data is the estimated Hurst parameter $H$. In case of a selfsimilar process, the zone should be located between two lines with slopes $1 / 2$ and 1 .

For the variance-time plot 8$]$, we plot $\log \left(\operatorname{var}\left(X_{k}^{(m)}\right)\right)$ vs. $\log (m)$ with a least-square fit for the plotted points (small values of $m$ are ignored). The process is self-similar if the estimated asymptotic slope $\hat{\beta}$ is between -1 and 0 . The Hurst parameter can then be estimated as $\hat{H}=1-\hat{\beta} / 2$.

\section{B. Telus Mobility traffic trace}

Traffic data were collected from the Telus Mobility CDPD network, located in downtown Vancouver area, from 14:56:37.56 to $15: 24: 46.88$ on June 12, 1998. Trace properties are given in Table I. The trace consisted of Mobile Data Link Layer Protocol (MDLP) frames. CDPD network subscribers used both TCP and UDP over IP protocols. The channel capacity was $19.2 \mathrm{kbps}$. During the time period when these data were collected, 10 mobile end systems appeared in the cell. Some were more and some were less active. For our statistical analysis, we used the aggregated traffic (the total input traffic to the mobile data base station) and selected only a 20 minute interval.

Table I. Telus Mobility CDPD network traffic trace.

\begin{tabular}{|c|c|c|c|c|}
\hline Duration & $\begin{array}{c}\text { Number of } \\
\text { link layer } \\
\text { frames }\end{array}$ & $\begin{array}{c}\text { Number of } \\
\text { bytes }\end{array}$ & $\begin{array}{c}\text { Average } \\
\text { traffic } \\
\text { load }\end{array}$ & $\begin{array}{c}\text { Network } \\
\text { utilization }\end{array}$ \\
\hline 20 minutes & 1,281 & 152,439 & 1,016 bps & $5.29 \%$ \\
\hline
\end{tabular}

Fig. 1 shows the R/S plot (top) and the variance-time plot (bottom). The Hurst parameter estimated from the slope in R/S plot is $\hat{H} \approx 0.80$. In Fig. 1 (bottom), the variance-time curve, which has been normalized by the corresponding sample variance, shows an asymptotic slope that is distinctly different from -1 (dotted line) and is estimated to be approximately
-0.2 , resulting in an estimate $\hat{H} \approx 0.90$ of the Hurst parameter. Unfortunately, the trace was too short for a more definite statement regarding its self-similarity.

These two graphical methods suggest that the traffic sequence is self-similar, with a self-similarity parameter $\hat{H} \geq 0.80$. According to [12], if $H \geq 0.60$, the data trace can be considered self-similar even if the trace has some degree of non-stationarity.
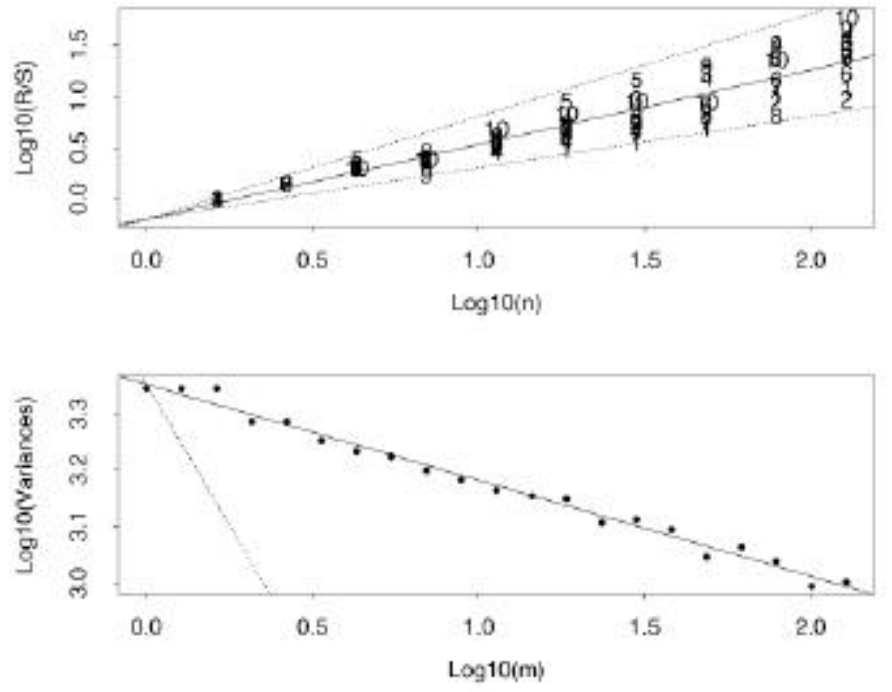

Fig. 1. Test for self-similarity: R/S plot (top) and variance-time plot (bottom).

\section{SIMULATION OF THE CDPD NETWORK}

\section{A. CDPD protocol}

CDPD [1, 14] is a standard protocol stack developed for mobile data networks. It is a multiple access protocol in which stations that want to transmit data into the network must compete for access in the shared communications medium. Although it shares some characteristics with more familiar multiple access protocols such as Ethernet (IEEE 802.3), CDPD differs from other multiple access protocols in two significant aspects: the wireless transmission medium and the mechanism for collision detection.

In most networks, the data transmission medium is either a wireline or a fiber-optic cable. In mobile data networks, radios transmit the data over the air-interface. This interface, in contrast to wires or fiber-optics, is more susceptible to bit errors introduced into the transmitted data. A typical and fairly static bit error rate (BER) for wired and fiber-optic networks is $10^{-10}$. The wireless BER is rather dynamic and can be as high as $10^{-2}$, or even higher [7]. This highly dynamic environment presents a unique challenge to the implementation of CDPD networks.

A second difference between CDPD and other multiple access protocols is in collision detection. In wired networks, stations 
can listen to their own transmissions. If another station is transmitting at the same time, stations will detect that a collision has occurred. When using radios to transmit data, mobile end systems (M-ES's) cannot reliably detect that a collision has occurred, and, therefore, must rely on the mobile data base station (MDBS) and the forward channel (from MDBS to M-ES) to determine whether the transmission was successful.

\section{B. OPNET model}

The topology of a simple CDPD network is shown in Fig. 2. Mobile end station (M-ES) is connected to the backbone network through the mobile data base station (MDBS). The network also includes a mobile data intermediate system (MDIS) (also called a mobile router) and several fixed end stations (F-ES) connected to the wired backbone network.

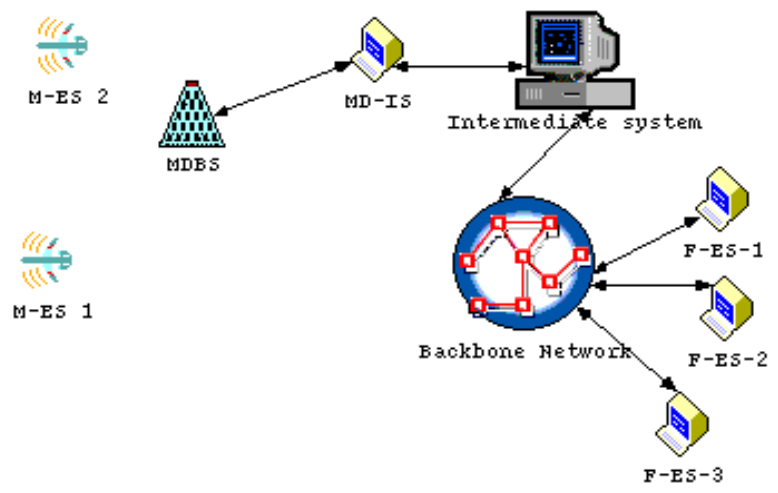

Fig. 2. Topology of a simple CDPD network.

We only modeled the wireless connection between M-ES and MDBS, i.e., the Media Access Control (MAC) layer of the CDPD protocol. In the M-ES node model in our simulations, we implemented a queueing system with the following characteristics: first-in-first-out (FIFO) queueing discipline, infinite buffer size, and arrivals that can be taken either from a genuine CDPD network traffic trace (trace-driven simulation), or from an ideal packet generator built in OPNET.

\section{Modeling of traffic sources}

In the absence of better wireless traffic models, we used tracedriven simulations and incorporated the collected CDPD network traffic trace into the network model. This was achieved by creating a process model, where collected traffic trace was read into the model from a file containing measured inter-arrival times and frame sizes. The underlying assumption in engineering practice, also used in our research work, is that the traffic environment is stationary over a range of time scales. While this assumption is not always satisfied in practice, it appears to be a reasonable hypothesis for the trace we have used.
In our simulation experiments, we also used input traces created by bursty ON/OFF traffic source models, and traces created from a process with exponentially distributed interarrival times (Poisson process). The ON/OFF model [13] generates a statistical process with the same mean as the genuine trace collected from the Telus Mobility CDPD network. Adjustment of the traffic burstiness is achieved by modifying the duration of the $\mathrm{ON}$ and OFF periods. In this model, three parameters are essential in determining the level of burstiness of the generated traffic: the mean duration of the $\mathrm{ON}$ state, the mean duration of the OFF state, and the number of frames sent during one ON period. In our simulations, durations of the ON periods and the OFF periods are exponential with means $a$ and $b$, respectively. Smaller values of $a$ indicate more bursty traffic. The number of frames generated during the $\mathrm{ON}$ period is 11 , obtained as a ceiling of a ratio of the total number of frames $(1,281)$ over the duration of the trace $(20 \mathrm{~min})$. It is calculated based on the mean rate of the traffic trace (1.067 packets/sec) and the duration of the ON/OFF cycle (10 seconds). The packet size in the ON/OFF traffic model is chosen to be 127 bytes, calculated to match the traffic load of the collected traffic trace $(1,016 \mathrm{bps})$.

All three traffic sources used in our study (actual trace, traces generated by ON/OFF models, and traces generated by Poisson arrival process) have the same traffic mean, and thus produce comparable traffic loads on the network. Therefore, we were able to compare the queueing performance of the CDPD network with various input traffic traces.

\section{SIMULATION RESULTS}

We performed a series of simulation experiments to demonstrate the practical significance of self-similarity in the queueing performance of a CDPD network. We show that using traditional queueing approximations to select CDPD network operating parameters may lead to overestimating network performance.

\section{A. Simulations of M-ES queueing delays}

The queueing delays at the M-ES buffer with three different traffic types are shown in Fig. 3. We plot the average queueing delays vs. link utilization for the actual CDPD traffic trace and for the traffic traces generated by the ON/OFF and Poisson sources. We varied the link utilization by both increasing the number of users (M-ES's) and by decreasing the link capacity in OPNET model that was equivalent to increasing the traffic load of a single user.

As can be seen from Fig. 3, the average queueing delay obtained with the genuine trace differs from the delay predicted by the Poisson (short-range dependent) arrival process. The burstiness of the input traffic trace was the feature that contributed to the sharp rise in delays at relatively high utilizations. The disparity between graphs A and E indicates that the short-range dependent traffic source model will greatly underestimate queueing delays at both moderate and high link utilizations. Our simulations also suggest that, although bursty ON/OFF traffic model (graph B) can emulate the genuine 
network traffic trace better than the Poisson model, this traffic model with a single distribution cannot adequately capture the traffic burstiness. The queueing delays corresponding to traces of lower burstiness is shown in graphs C and D of Fig. 3 .

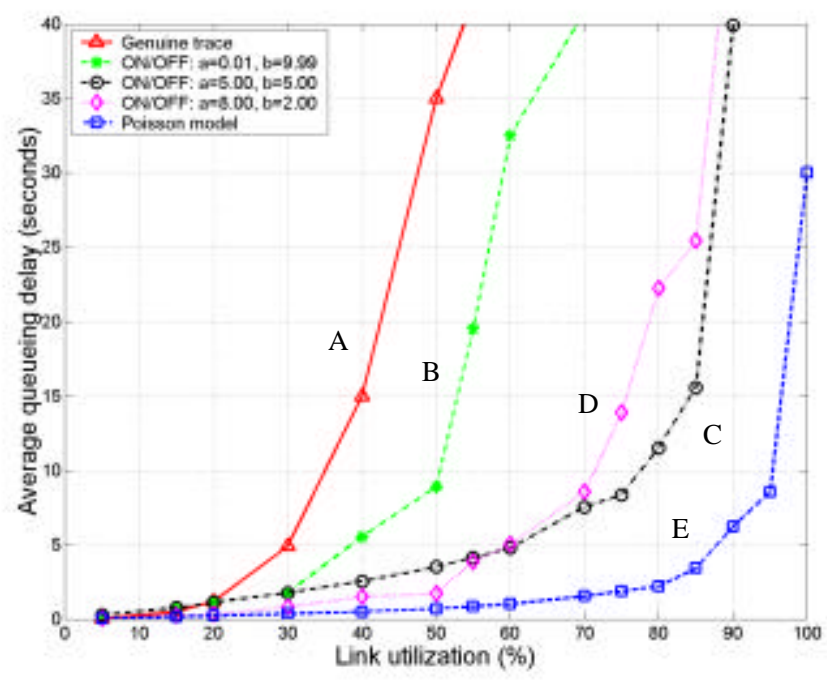

Fig. 3. Average queueing delay vs. link utilization plots for genuine traffic trace (graph A), traffic generated by ON/OFF models (graphs B, C, and D), and traffic generated by the traditional Poisson model (graph E). For ON/OFF sources the parameters are: (B) $\mathrm{a}=0.01 \mathrm{sec}$ and $\mathrm{b}=9.99 \mathrm{sec} ;(\mathrm{C}) \mathrm{a}=5.00$ sec and $b=5.00 \mathrm{sec} ;(D) a=8.00 \mathrm{sec}$ and $b=2.00 \mathrm{sec}$.

The queueing delay graphs obtained by using the actual CDPD trace (graph A) and the most bursty ON/OFF model (graph B) differ by approximately $10 \%$. These differences arise because the ON/OFF source model employs exponential distribution of its ON and OFF periods. There are also ON/OFF models where the ON and OFF periods may employ more general distributions with finite variance and short-range dependence. Nevertheless, it has been recently recognized that multiplexing a large number of ON/OFF traffic sources results in aggregate traffic that is inconsistent with traffic measurements from actual networks [16].

\section{B. M-ES buffer overflow probabilities}

While in the study of average queueing delays we chose infinite size buffers, in this section we select a finite M-ES buffer size and we investigate two relationships: buffer overflow probability vs. buffer size and vs. link utilization, respectively.

First, we investigate the relationship between buffer overflow probability and the buffer size. The link utilization is kept constant at $40 \%$. The buffer overflow probabilities with genuine traffic (A) and Poisson traffic (B) are shown in Fig. 4. A linear region on the semi-log plot indicates that the probability of buffer overflow is exponential with a constant decay. The buffer overflow probability resulting from Poisson traffic (B) is exponential and decreases much faster than the buffer overflow probability resulting from the genuine traffic trace (A). Other short-range dependent models with constant or exponential service times also exhibit this fast-decaying behavior.

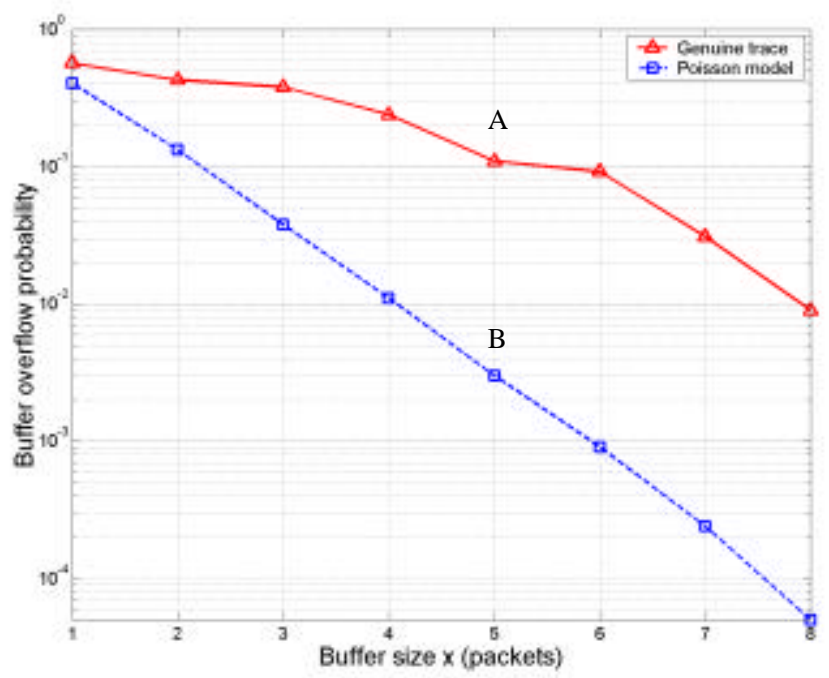

Fig. 4. Buffer overflow probability vs. buffer size plots for (A) genuine traffic trace and (B) Poisson arrival traffic source.

In our second study we investigate the relationship between buffer overflow probability and link utilization. We varied the link utilization in the same manner as in our simulations of MES queueing delays. A plot of $P(q>x)$, where $q$ is the queue length, is shown in Fig. 5. The buffer size $x$ is set to five packets. The larger buffer overflow probability in Fig. 5, compared to Fig. 4, is the result of using larger number of users to achieve higher link utilizations. Fig. 5 illustrates that even at relatively low link utilizations $(\sim 20 \%)$, the buffer overflow probability obtained by using the genuine traffic trace is noticeably larger than by using a Poisson traffic source model. The ON/OFF traffic model, as expected, predicts the buffer overflow more accurately than the Poisson model.

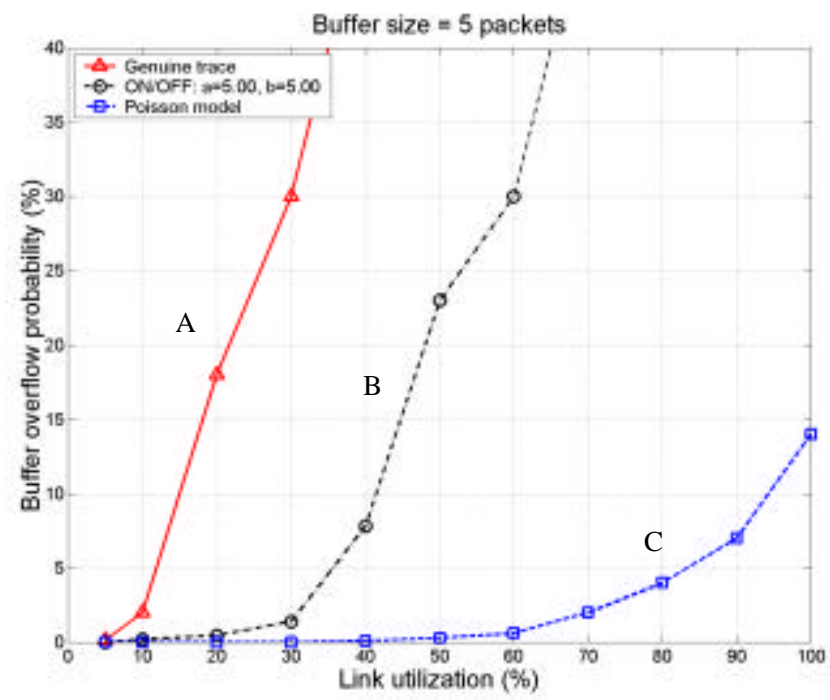

Fig. 5. Buffer overflow probability vs. link utilization plots for (A) genuine traffic trace; (B) ON/OFF source model with a = $5.00 \mathrm{sec}$ and $\mathrm{b}=5.00 \mathrm{sec}$; (C) Poisson arrival traffic source. 
The results of our simulations imply the importance of capturing long-range characteristics of traffic models in wireless networks, and indicate "the end of simple traffic models" $[9,10]$. Self-similarity has emerged as one of the most important characteristics that needs to be captured by network traffic models $[8,15,16]$. Traditional traffic models provide limited insight into the true nature of genuine traffic data, often fail to capture the properties of genuine traffic, have no meaningful physical interpretation, and have limited value in the engineering of future networks. In contrast, characteristics observed in measured traffic data can be captured with longrange dependent (self-similar) processes described with one parameter (Hurst parameter) [2]. These processes can also be generated by fractional Gaussian noise (FGN) and by fractionally differenced autoregressive integrated movingaverage (FARIMA) models. For example, LAN traffic can be successfully modeled using an FGN process with three parameters: mean, variance, and the Hurst parameter [15]. A FARIMA process with four or five parameters was found to describe VBR video traffic with reasonable accuracy [5].

\section{CONCLUSIONS}

In this paper we analyzed traffic collected from the Telus Mobility CDPD network. Our preliminary results suggest that this wireless data traffic tends to have a self-similar behavior, and that it is statistically different from traffic generated by traditional traffic models. Nevertheless, our traffic trace was too short (20 minutes long) to warrant a more definite conclusion.

We used trace-driven OPNET simulations to demonstrate that long-range dependence is an important traffic characteristic in wireless networks, and, if ignored, may result in overly optimistic performance predictions and inadequate network resource allocations. Our simulation results show that the queueing delays obtained with the genuine traffic trace differ from the queueing delays predicted by short-range dependent traffic source models (ON/OFF and Poisson arrival processes). In the case of moderate and high link utilizations, short-range dependent traffic models underestimate queueing delays. Thus, genuine traffic traces produce longer queues and require larger buffers in the network's elements. Genuine traffic traces also cause higher buffer overflow probabilities than the conventional traffic models. This may require lower wireless link utilizations in order to avoid undesired loss rates.

\section{ACKNOWLEDGMENTS}

We thank Bruce Chen from Simon Fraser University for improving the OPNET simulations. We also acknowledge the assistance of Telus Mobility in providing traffic traces, and to OPNET Inc. for the access to their simulation tools.
1. J. Agosta and T. Russell, CDPD: Cellular Digital Packet Data Standards and Technology. Reading, MA: McGrawHill, 1996.

2. J. Beran, Statistics for Long-Memory Processes (Monographs on Statistics and Applied Probability). London: Chapman and Hall, 1994.

3. M. E. Crovella and A. Bestavros, "Self-similarity in world wide web traffic: evidence and possible causes," IEEE/ACM Trans. Networking, vol. 5, no. 6, pp. 835 846, Dec. 1997.

4. D. E. Duffy, A. A. McIntosh, M. Rosenstein and W. Willinger, "Statistical analysis of CCSN/SS7 traffic data from working CCS subnetworks," IEEE J. Select. Areas Commun., vol. 12, no. 3, pp. 544 - 551, Apr. 1994.

5. M. Garrett and W. Willinger, "Analysis, modeling and generation of self-similar VBR video traffic," in Proc. ACM SIGCOMM'94, London, U.K., Aug. 1994, pp. 269 280.

6. I. Katzela, Modeling and Simulating Communication Networks: A Hands-On Approach Using OPNET. Upper Saddle River, NJ: Prentice Hall, 1999.

7. W. C. Y. Lee, Mobile Cellular Telecommunications Systems. New York, NY: McGraw-Hill, 1989.

8. W. Leland, M. Taqqu, W. Willinger, and D. Wilson, "On the self-similar nature of Ethernet traffic (extended version)," IEEE/ACM Trans. Networking, vol. 2, no. 1, pp. 1 - 15, Feb. 1994.

9. C. Partridge, "The end of simple traffic models," IEEE Network, vol. 7, no. 5, p. 3, Sept. 1993.

10. V. Paxson, "Empirically derived analytic models of widearea TCP connections," IEEE/ACM Trans. Networking, vol. 2, pp. 316 - 336, Aug. 1994.

11. V. Paxson and S. Floyd, "Wide-area traffic: the failure of Poisson modeling," in Proc. ACM SIGCOMM'94, London, U.K., Aug. 1994, pp. 257 -268.

12. M. Roughan and D. Veitch, "Measuring long-range dependence under changing traffic conditions," in Proc. IEEE INFOCOM'99, New York, NY, Mar. 1999, pp. 338 $-341$.

13. K. Sohraby, "On the theory of general ON-OFF sources with applications in high speed networks," in Proc. IEEE INFOCOM'93, San Francisco, CA, Apr. 1993, pp. 401 410.

14. M. Sreetharan and R. Kumar, Cellular Digital Packet Data. Norwood, MA: Artech House, 1996.

15. W. Willinger, M. S. Taqqu, W. E. Leland, and D. V. Wilson, "Self-similarity in high-speed packet traffic: analysis and modeling of Ethernet traffic measurements," Statistical Science, vol. 10, no. 1, pp. 67 - 85, 1995.

16. W. Willinger, M. S. Taqqu, R. Sherman, and D. V. Wilson, "Self-similarity through high-variability: statistical analysis of Ethernet LAN traffic at the source level,' IEEE/ACM Trans. Networking, vol. 5, pp. 71 - 86, Feb. 1997. 